\title{
Quantification of the Prediction Requirements in Reactive Control of Wave Energy Converters *
}

\author{
Francesco Fusco* John Ringwood* \\ * Center for Ocean Energy Research \\ National University of Ireland Maynooth, Ireland \\ (e-mail: francesco.fusco@eeng.nuim.ie)
}

\begin{abstract}
Optimal reactive control for maximum ocean wave power absorption from Wave Energy Converters (WECs) consisting of oscillating systems, is based on the principle of tuning their oscillation so that it is in resonance with the excitation force produced by the incident waves. Reactive control, however, is non-causal and cannot be implemented in real time. This paper analyses the prediction requirements of one possible solution, where predictions of the excitation force are utilised to resolve the non-causality. The study is focused on the analysis of the required forecasting horizon against the achievable prediction. Also, through the aid of numerical simulations of a number of specific systems over several wave conditions, a link is found between some fundamental properties of the system and the prediction requirements.
\end{abstract}

Keywords: Wave energy, impedance matching, time series forecasting.

\section{INTRODUCTION}

The efficiency of Wave Energy Converters (WECs), consisting of oscillating systems, can be significantly increased through an automatic control that tunes its oscillations to the incident wave elevation, in such a way to improve the power transfer from the ocean to the system. The analytical optimal solution, for the maximisation of the energy extraction, requires the system to be in resonance with the wave force or pressure (Falnes, 2002), and it is termed reactive control, or complex-conjugate control for its analogy in electrical systems. Alternative sub-optimal control solutions, formulated as constrained optimisation problems, have also been proposed, where the limitation imposed by the physics of the system (e.g. amplitude of motion or velocity, applicable forces), ignored by reactive control, are also taken into account. In particular, some of these alternatives are latching (Babarit and Clement, 2006), where the oscillation in the system is delayed so to be in phase with the excitation from the waves, and Model Predictive Control (MPC) (Bacelli et al., 2009; Cretel et al., 2010; Hals et al., 2011), which handles the use of constraints.

The effectiveness of the different real-time control strategies depends, among the others, on the possibility to predict the future wave elevation or wave excitation force acting on the system at least for a few seconds into the future (Falnes, 2007; Babarit and Clement, 2006; Bacelli et al., 2009). Short-term wave forecasting was studied either with a deterministic approach (Belmont et al., 2006; Tedd and Frigaard, 2007; Van Den Boom, 2009), and as a purely stochastic univariate time series problem (Fusco

* This work was funded by the Irish Research Council of Science, Engineering and Technologies (IRCSET) under the Embark Initiative. and Ringwood, 2010). In the latter, in particular, it is demonstrated how accurate predictions of the swell can be achieved with simple autoregressive (AR) models for more than one mean wave period ahead.

This paper is focused on the evaluation of the effect that future knowledge of the excitation force has on the performance of reactive control applied to a simple oscillating system in one degree of freedom. Based on the preliminary study reported in Fusco et al. (2010), a methodology is presented, that attempts to link the fundamental properties of the conversion system (radiation and excitation in particular) to how much the system itself may be demanding in terms of prediction as well as to the predictability of the wave excitation force.

After an introduction to reactive control, in section 2, the methodology for the analysis of the prediction requirements is proposed in section 3. Results of numerical simulation of a number of floating systems over a variety of real wave data are then presented in section 4 . Conclusive remarks are outlined in section 5 .

\section{REACTIVE CONTROL}

A generic floating body, in a single degree of freedom, is considered, as simply schematised in Fig. 1. The system, under the influence of the excitation force $f_{e x}(t)$ from incident waves, oscillates according to the following dynamics:

$$
\begin{array}{rl}
m \dot{v}(t)+\int_{0}^{t} z(t-\tau) v(\tau) d \tau+K_{f} & v(t)+K_{s} x(t) \\
& =f_{e x}(t)+f_{u}(t) .
\end{array}
$$

Here, $m$ is the mass of the body, $v(t)$ and $x(t)$ its velocity and position, $z(t)$ is the impulse response of the radiation damping force, $K_{f}$ is a constant modelling the friction, $K_{s}$ 


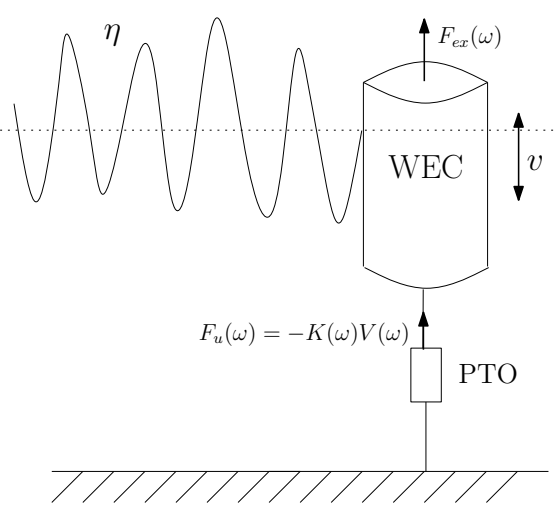

Fig. 1. Heaving body oscillating with respect to sea bottom under the influence of an incident wave.

is the buoyancy coefficient and $f_{u}(t)$ is a controllable force, that can be produced through the power take-off (PTO) mechanism. Zero initial conditions, namely $v(0)=x(0)=$ 0 , are assumed.

In the frequency domain, equation (1) becomes:

$$
Z_{i}(\omega) V(\omega)=F_{e x}(\omega)+F_{u}(\omega),
$$

where the intrinsic mechanical impedance $Z_{i}(\omega)$ is defined as:

$$
Z_{i}(\omega) \triangleq \jmath m \omega+Z(\omega)+K_{f}+\frac{K_{s}}{\jmath \omega}
$$

In (3), the radiation impedance $Z(\omega)$ was introduced, which is conveniently expressed in terms of radiation damping, $B(\omega)$, and added mass, $M(\omega)$, as:

$$
Z(\omega) \triangleq B(\omega)+\jmath \omega M_{a}(\omega)
$$

Note that $Z(\omega)$ can be defined as the Fourier transform of the radiation force kernel, $z(t)$, but only in a generalised sense, as $M_{a}(\omega)$ does not, in general, vanish in the limit $\omega \rightarrow+\infty$ (Falnes, 2002). This singularity is usually made explicit through a constant $M_{a}(\infty)$, the added mass at infinite frequency.

From (3) and (4), the intrinsic mechanical impedance can finally be written as:

$$
Z_{i}(\omega)=B(\omega)+K_{f}+\jmath \omega\left[m+M_{a}(\omega)-\frac{K_{s}}{\omega^{2}}\right],
$$

The external excitation from the waves, $f_{e x}(t)$, is determined by the excitation transfer function $H_{e x}(\omega)$ :

$$
F_{e x}(\omega)=H_{e x}(\omega) \Xi(\omega),
$$

where $\Xi(\omega)=\mathscr{F}\{\eta(t)\}$.

Given the system in equations (1) and (2), the control objective, for a WEC, is to choose a load force, $F_{u}(\omega)=$ $\mathscr{F}\left\{f_{u}(t)\right\}$, such that the mechanical energy transfer from the waves to the load is maximised. The PTO is left unspecified at this stage, the only assumption being that it is able to provide a force of the form

$$
F_{u}(\omega)=-K(\omega) V(\omega),
$$

where $K(\omega)$ is the load impedance. The control strategy can be represented as in the block scheme of Fig. 2.

The average power absorbed at the load is the time integral of the product of the load force and the system velocity, which can be also expressed in the frequency domain, in terms of cross-spectral power densities, $\phi_{f_{u} v}(\omega)$, of the two real signal $f_{u}(t)$ and $v(t)$ (MacMartin and Hall, 1991):

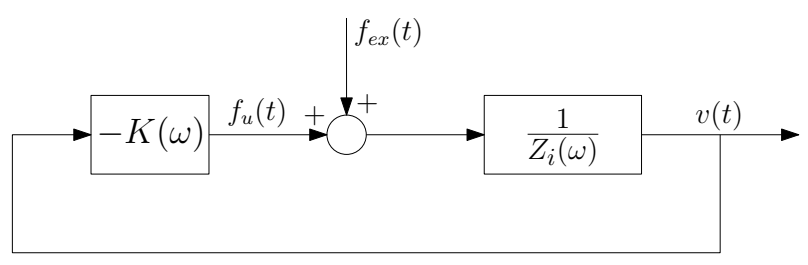

Fig. 2. Block diagram of reactive control

$$
\begin{aligned}
P_{u}=-\lim _{T \rightarrow \infty} \frac{1}{2 T} & \int_{-T}^{T} f_{u}(t) v(t) d t= \\
& -\frac{1}{2 \pi} \int_{0}^{\infty}\left[\phi_{f_{u} v}(\omega)+\phi_{v f_{u}}(\omega)\right] d \omega,
\end{aligned}
$$

The integrand of the right hand side (with the minus sign) represents the average power flow going out of the system through the load as a function of the frequency, so that the average wave power absorbed at each frequency can be defined as:

$$
P_{u}(\omega)=-\left[\phi_{f_{u} v}(\omega)+\phi_{v f_{u}}(\omega)\right]
$$

Using (2) and (7), it is possible to express $P_{u}(\omega)$ in terms of the spectral power density of the excitation force, $\phi_{e x}(\omega)$ :

$$
P_{u}(\omega)=\frac{K+K^{*}}{\left(Z_{i}+K\right)\left(Z_{i}+K\right)^{*}} \phi_{e x}
$$

where the explicit dependance on the frequency has been dropped for clarity. Note that the ${ }^{*}$ symbol denotes the complex-conjugate operation.

Maximisation of (10) gives the optimal load impedance:

$$
P_{u}(\omega)=\max \quad \Leftrightarrow \quad K=K_{o p t}=Z_{i}^{*},
$$

where the maximum of the average absorbed power is:

$$
P_{u, o p t}(\omega)=\frac{\phi_{e x}(\omega)}{2 B(\omega)+2 K_{f}} .
$$

$P_{u, o p t}(\omega)$ can be shown to be half the excitation power and equal to the sum of the radiated power and the power due to friction (Falnes, 2002).

The control law in (11) is termed, in the wave energy literature, complex-conjugate control because the optimal load impedance has to match the complex-conjugate of the intrinsic impedance (note the analogy with electrical circuits). The name reactive control is also used, to highlight the presence of a reactive power and therefore the necessity to inject power into the system during part of the cycle. This requirement of a reversible power flow has major implications on the effective practical implementation of reactive control. Although maximum mechanical energy is ensured, in fact, losses in the bi-directional flow implemented by a non-ideal PTO mechanism may in fact result in a poor overall efficiency of the system, if one considers the useful (electrical) energy produced.

Under optimality condition (11), the velocity and the load force are related to the excitation force through the following relations:

$$
\begin{aligned}
V_{\text {opt }}(\omega) & =\frac{1}{2 B(\omega)+2 K_{f}} F_{e x}(\omega) \\
F_{u, o p t}(\omega) & =-Z_{i}^{*}(\omega) V(\omega)=-\frac{Z_{i}^{*}(\omega)}{2 B(\omega)+K_{f}} F_{e x}(\omega)
\end{aligned}
$$


Conditions (14) and (13), as well as the optimal compensator in (11), however, are non-causal and cannot be implemented (Falnes, 2002; MacMartin and Hall, 1991). Prediction of the excitation force or velocity of the system, though, could allow for a suboptimal realisation. While this paper is focused on a solution based on prediction of the excitation force, a possibility to implement reactive control, where condition (14) is approximated through the prediction of the oscillation velocity of the system, was proposed by Korde (2000).

\section{METHODOLOGY}

Either of the reactive control optimality conditions (13) and (14), which apply when the WEC is in the state of absorbing the maximum energy from the waves, can be utilised in order to provide the reference velocity, $v(t)$, or PTO force, $f_{u}(t)$, that shall be imposed on the system, eventually through one or more lower level control loops.

For the present study, it is supposed that an optimal reference velocity is generated from the non-causal relation (13), using future values of the excitation force. Note that the control variable of the system is the PTO force, $f_{u}(t)$, so that an additional lower level loop imposing the reference velocity through the force is required. The force is then realised in the PTO mechanism by means of a further low level control loop. The whole control strategy is depicted in Fig. 3.

The study of the prediction requirement is divided into two main sub-problems. First, in section 3.1, the noncausality of the optimal transfer function, relating the excitation force to the oscillating velocity, is analysed and linked to fundamental properties of the system. In particular, a quantification of the effects that knowledge of the future excitation force, over a limited time horizon, has on the control performance (absorbed wave power), is proposed. Then, in section 3.2, the ability to predict the excitation force is studied and, again, linked to a fundamental property of the WEC.

\subsection{Effects of future knowledge of the excitation force on absorbed power}

The optimal reference velocity for the WEC, from Fig. 3 and eq. (13), is calculated, in the time-domain, as:

$$
v_{\text {opt }}(t)=\int_{-\infty}^{t} h_{\text {opt }}(\tau) f_{\text {ex }}(t-\tau) d \tau
$$

where

$$
h_{o p t}(t)=\mathscr{F}^{-1}\left\{\frac{1}{2 B(\omega)+2 K_{f}}\right\}=\mathscr{F}^{-1}\left\{H_{o p t}(\omega)\right\}
$$

When $\omega \rightarrow \infty$, the radiation resistance $B(\omega)$ goes to zero and therefore $H_{o p t}(\omega)$ tends to the constant value $1 / 2 K_{f}$. This means that the transform relationship in (16) is only valid in a generalised sense and, more properly,:

$$
h_{o p t}(t)=\frac{1}{2 K_{f}} \delta(t)+\mathscr{F}^{-1}\left\{H_{o p t}(\omega)-\frac{1}{2 K_{f}}\right\},
$$

where the inverse Fourier transform in the right-hand term is now well defined. Note that $B(\omega)$, in general, is only known numerically for some frequencies, and $h_{\text {opt }}(t)$ need to be derived numerically with the inverse discrete Fourier transform, where the sampling frequency is determined by the maximum frequency for which $B(\omega)$ is available. Zeropadding can be applied to obtain any desired sampling frequency with no loss of accuracy, once the singularity is removed, as in (17). Note that, if no friction is considered, the Fourier inversion would be much more complicated.

As previously stated, the optimal velocity can only be calculated from (15) if the excitation force is known infinitely far into the future. In practice, only knowledge over a limited time horizon $L$ can be assumed, which will determine an imperfect reference and non-optimal performance of the system:

$$
v_{\text {ref }}^{L}(t)=\int_{-L}^{t} h_{o p t}(\tau) f_{e x}(t-\tau) d \tau \approx v_{o p t}(t) .
$$

For a given $L$, the performance of the control can be measured in terms of Relative Capture Width (RCW), defined as the average absorbed power divided by the average wave power available over the physical width, $D$, of the device:

$$
R C W(L)=\frac{P_{u}(L)}{P_{w} \cdot D}
$$

Here, we explicitly include the dependance on the future horizon $L$. The average absorbed power, $P_{u}(L)$, can be directly calculated from (8), while the average wave power per meter of wave front, $P_{w}$, is determined as usual:

$$
P_{w}=\frac{1}{2} \rho g^{2} \int_{0}^{+\infty} \frac{S_{\eta \eta}(\omega)^{*}}{\omega} d \omega,
$$

where $S_{\eta \eta}(\omega)=\Xi(\omega) \Xi^{*}(\omega)$ is the wave spectrum. Also, deep water is assumed, that is $k h>>1$, where $k$ is the wave number and $h$ is the water depth.

Intuitively, we can expect $R C W(L)$ to increase with $L$ but only up to a critical future horizon, beyond which the power gain from considering extra future information is negligible. Such a critical horizon is related to the time interval after which the non-causal (but stable) impulse response $h_{\text {opt }}(t)$ is almost zero and, ultimately, to some fundamental property of the system's radiation resistance $B(\omega)$.

In order to determine such a basic property of the system, consider the transform pair $h_{o p t}(t)$ and $H_{o p t}(\omega)$ of Fig. 4. From well known relations between (stable) impulse response functions and correspondent transfer functions, the decay towards zero is dominated by an exponential connected to the dominant time constant of the system, that is the pole with the lowest frequency. If we consider $H_{\text {opt }}(\omega)$ as a transfer function, the lowest frequency pole may be thought of as the cut-off frequency of the first lobe with a low-pass-filter like characteristic, as indicated in Fig. 4. It may be expected, therefore, that the lower the frequency of this dominant pole, the longer the decay of $h_{\text {opt }}(t)$ and therefore the longer the future horizon $L$ for which a close approximation of the optimal power capture is obtained. The dominant time constant, thus identified, will be referred to as $\tau_{1}$ in the remainder of the paper.

\subsection{Predictability of the excitation force}

In practice, the wave excitation force needs to be predicted. As mentioned in section 1, several studies have been pro- 


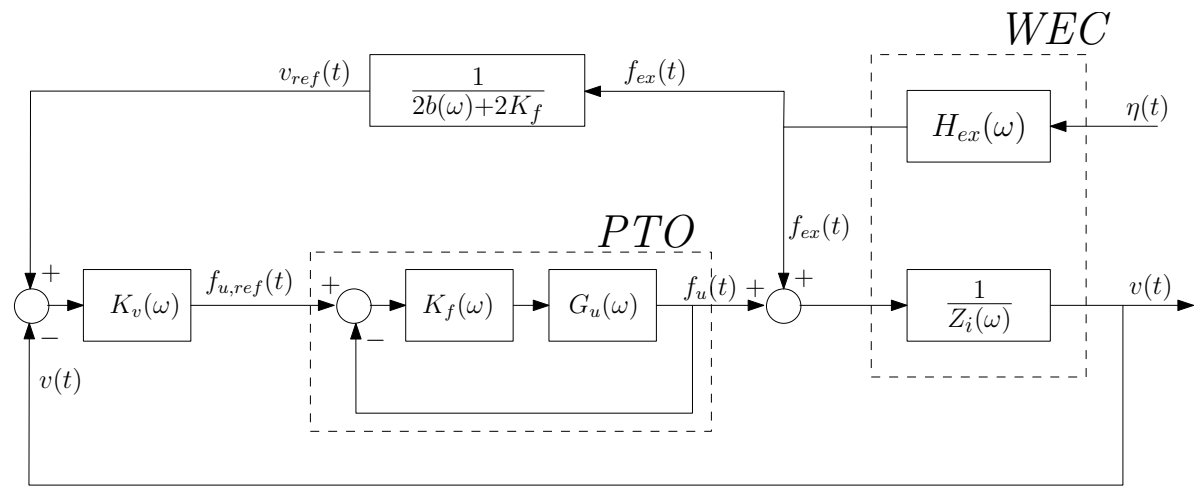

Fig. 3. Realisation of reactive control imposing the optimal velocity on the system. Two additional lower level control loops are required to decide the appropriate load force and then realise it on the power take-off (PTO).
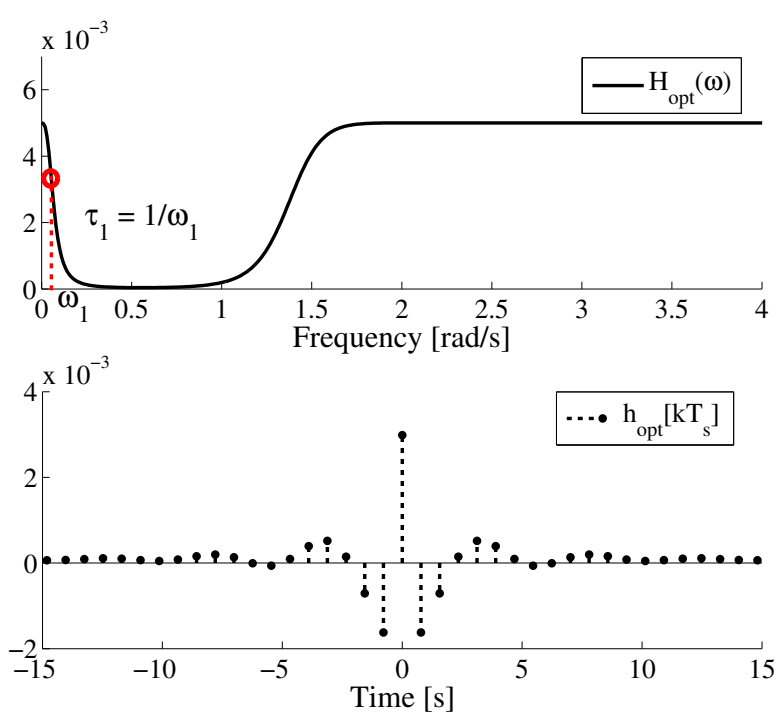

Fig. 4. $H_{o p t}(\omega)$ and inverse discrete Fourier transform $h_{\text {opt }}(t)$ for a floating cylinder with radius $5 \mathrm{~m}$, draught of $20 \mathrm{~m}$ and mass of $1.62 \times 10^{6} \mathrm{Kg}$. Sampling frequency is $f_{s}=2.56 \mathrm{~Hz}$ (Nyquist frequency $4.02 \mathrm{rad} / \mathrm{s}$ ).

posed for the problem of short-term wave forecasting, and they can be extended to the prediction of the excitation force, which is the wave elevation force low-pass filtered by the system's excitation transfer function $H_{e x}(\omega)$, as from (6). For a given sea state, the properties of $H_{e x}(\omega)$ have an influence on the predictability of the excitation force. The purpose of this study is to give a quantification of the achievable forecasting horizon (within a certain accuracy of the prediction), possibly to connect it to a fundamental property of the system, and to compare it against the required prediction, determined as from section 3.1.

AR models are adopted here, whose identification and estimation are operated accordingly to the methodology presented in Fusco and Ringwood (2010). In particular, at instant $k$, the $l$-step ahead prediction, $\hat{f}_{\text {ex }}(k+l \mid k)$, is calculated as:

$$
\hat{f}_{e x}(k+l \mid k)=\sum_{i=1}^{n} a_{i}(k) \hat{f}_{e x}(k+l-i \mid k),
$$

where $a_{i}, i=1, \ldots n$ are the coefficients of the AR model, of order $n$, and, obviously, $\hat{f}_{e x}(k+l-i \mid k)=f_{e x}(k+l-i)$ if $k+l-i \leq k$ (information acquired, no need of prediction).
The performance of the prediction algorithm is measured in terms of the following index of goodness-of-fit:

$$
\mathcal{F}(l)=\left(1-\frac{\sqrt{\sum_{k=1}^{T}\left[f_{e x}(k+l)-\hat{f_{e x}}(k+l \mid k)\right]^{2}}}{\sqrt{\sum_{k=1}^{T} f_{e x}(k)^{2}}}\right) \cdot 100,
$$

where $T$ is the time of simulation.

As discussed in Fusco et al. (2010), the ability to predict the excitation force acting on a system is connected to the filtering capability of the transfer function $H_{e x}(\omega)$. The latter has a low-pass characteristic, so that its cut-off frequency is the same as its bandwidth. As a consequence systems with narrower excitation bandwidth will experience excitation forces from waves at lower frequencies, that, in general, have better predictability properties (Fusco and Ringwood, 2010). Ultimately we would expect that the achievable prediction horizon for the excitation force is inversely proportional to the bandwidth of $H_{e x}(\omega)$.

\section{RESULTS}

The methodology outlined in section 3 is tested over a range of bottom-referenced floating cylinders (Fig. 1), whose characteristics are shown in Table 1. For each cylinder, the value of the time constant $\tau_{1}$, which is expected to influence the prediction requirements, and the bandwidth of $H_{e x}(\omega)$, namely $\Delta \omega_{e x}$, which should have an influence on the predictability, are also shown. Note how the value of $\tau_{1}$ is almost entirely influenced by the radius. A friction $K_{f}=100 \mathrm{Ns} / \mathrm{m}$ is also introduced, its absolute value not affecting the generality of the results. The excitation frequency response, $H_{e x}(\omega)$, and the radiation impedance, $Z(\omega)$, were calculated, for some frequencies, with the hydrodynamic software WAMIT Inc. (2008).

The systems are simulated, in the time domain, under several sea conditions. The wave data was provided by the Irish Marine Institute and comes from real measurements collected from a data buoy deployed off the West coast of Ireland, in the Belmullet wave energy test site, at approximately $54^{\circ} 130 \mathrm{~N} ; 10^{\circ} 80 \mathrm{~W}$. The data consists of two consecutive 30 minutes sets for each hour, sampled at $1.28 \mathrm{~Hz}$, covering the year 2010. Fig. 5 shows the distribution of peak frequency and significant wave height, to give an idea of the wave climate at the location. 


\begin{tabular}{c||crr||c|c|} 
cylinder & $R[m]$ & $h[m]$ & $m[K g]$ & $\Delta \omega_{\text {ex }}$ & $\tau_{1}[s]$ \\
\hline 1 & 3 & 4 & $0.93 \times 10^{5}$ & 1.03 & 7.42 \\
2 & 3 & 8 & $1.86 \times 10^{5}$ & 0.79 & 7.39 \\
3 & 3 & 12 & $2.80 \times 10^{5}$ & 0.66 & 7.35 \\
4 & 3 & 16 & $3.73 \times 10^{5}$ & 0.59 & 7.32 \\
5 & 3 & 20 & $4.66 \times 10^{5}$ & 0.53 & 7.27 \\
6 & 5 & 4 & $2.59 \times 10^{5}$ & 0.98 & 14.75 \\
7 & 5 & 8 & $5.18 \times 10^{5}$ & 0.75 & 14.73 \\
8 & 5 & 12 & $7.77 \times 10^{5}$ & 0.63 & 14.71 \\
9 & 5 & 16 & $10.40 \times 10^{5}$ & 0.56 & 14.68 \\
10 & 5 & 20 & $12.90 \times 10^{5}$ & 0.51 & 14.66 \\
11 & 7 & 4 & $5.07 \times 10^{5}$ & 0.95 & 23.09 \\
12 & 7 & 8 & $10.10 \times 10^{5}$ & 7.2 & 23.09 \\
13 & 7 & 12 & $15.20 \times 10^{5}$ & 0.62 & 23.09 \\
14 & 7 & 16 & $20.30 \times 10^{5}$ & 0.54 & 23.09 \\
15 & 7 & 20 & $25.40 \times 10^{5}$ & 0.50 & 23.09
\end{tabular}

Table 1 . Heaving cylinders analysed. $R$ : radius;

$h$ : draught at rest; $m$ : submerged mass at rest.

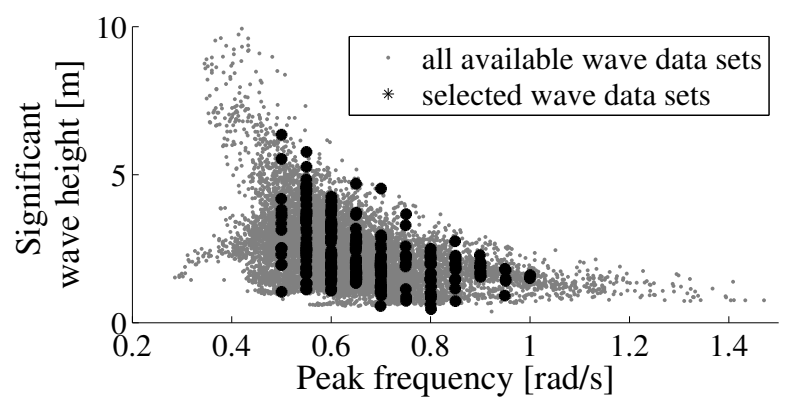

Fig. 5. Distribution of peak frequency, $\omega_{p}$, and significant wave height, $H_{s}$, of the data sets selected among the available ones, collected at Belmullet during the year 2010.

The performance of reactive control when a limited future horizon $L$ is considered, in the calculation of the reference velocity, is assessed with the methodology of section 3.1. Fig. 6 shows the behavior of $R C W(L)$ for three of the cylinders in Table 1, with same height but different radius. As expected, the $R C W(L)$ increases when more and more future information about the wave excitation force is included in the reference velocity calculation. More interestingly, the curves approach the maximum at different time horizons and, in particular, the prediction requirements are more demanding for systems with a larger time constant $\tau_{1}$ (bigger radius). While the maximum of the curve $R C W(L)$ depends on the specific sea state, its general behavior only depends on the systems properties, $h_{\text {opt }}(t)$ and the radiation resistance in particular.

In order to have a more general picture about the relation between prediction requirements and properties of the WEC, the behavior of $R C W(L)$ was calculated for each of the 15 cylinders over the selected sea states. For each system, the average value of the forecasting horizon $L$, required to obtain a $R C W(L) \geq 0$ was determined, and this is shown in Fig. 7 against the corresponding $\tau_{1}$ of the cylinder. The prediction requirements increase almost linearly with the dominant time constant of the function $h_{\text {opt }}(t)$. The standard deviation for each point is also shown as the length of a bar centered about the mean value (the circle). Its value is relatively small and this fact highlights the independence of the prediction requirements from the specific sea state. Note, from Fig. 7, that the height



Fig. 6. $R C W(L)$ obtained with three cylinders, over a specific sea state.

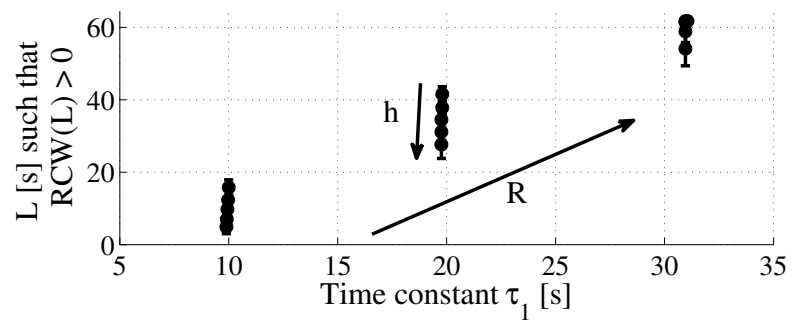

Fig. 7. Average prediction horizon required to obtain a $R C W \geq 0$, calculated for each cylinder over the selected data sets.



Fig. 8. Prediction accuracy, with AR models, of the excitation force acting on three different cylinders.

of the cylinder also has an influence on the forecasting horizon required for a positive $R C W$. In particular, for a given radius ( or $\tau_{1}$ ), when the height increases, the device becomes less demanding in terms of prediction. The main parameter, though, remains as $\tau_{1}$.

Regarding the prediction of the excitation force, based on the discussion in section 3.2, AR models are utilised (Fusco and Ringwood, 2010) and it is expected that, for a given sea state, systems with smaller excitation bandwidth, $\Delta \omega_{e x}$, experience a more predictable excitation force signal. In Fig. 8, the accuracy of the prediction, expressed through the quantity $\mathcal{F}(l)$ defined in $(22)$, is shown in detail for three different cylinders, for a particular sea state. Obviously, the accuracy of the prediction decreases with the prediction horizon, but, more interestingly, in the case of cylinders with smaller $\Delta \omega_{e x}$, the prediction is more accurate over a longer future horizon.

Again, a more general picture is offered, in Fig. 9, where, for each device, the average forecasting horizon below which the prediction has an accuracy of $\mathcal{F}(l) \geq 60 \%$, calculated over several sea states, is shown. The variance 


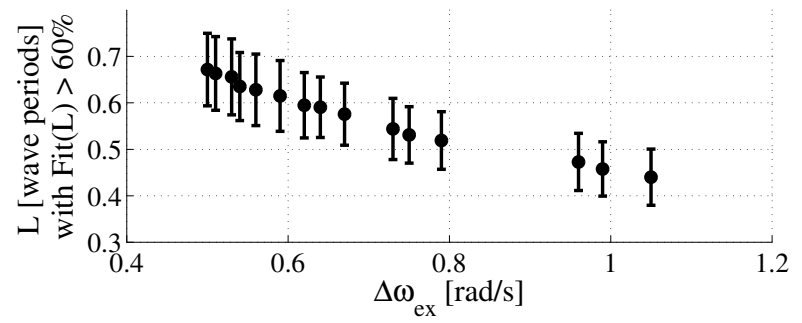

Fig. 9. Average prediction horizon achieved with an accuracy of $\mathcal{F} \geq 60 \%$, calculated for each cylinder over the selected data sets.

of each value is also shown through the length of a bar centered about the mean value. The prediction accuracy increases almost linearly as the bandwidth of the systems decreases. In most of the cases, more than half a wave period can be predicted with an accuracy above $60 \%$. Note, however, that the variance of such a prediction horizon, for each device, is quite significant as external parameters (wave properties) also have a strong influence on the prediction.

\section{CONCLUSION}

The prediction requirements of reactive control for maximum wave energy extraction from a Wave Energy Converter (WEC) in a single degree of freedom were discussed. In particular, we investigated the possibility of realising the non-causal relationship between the optimal oscillation velocity and the excitation force induced by the incident wave, using predictions of the excitation force itself.

The study of the prediction requirements was focused on two main properties: the forecasting horizon required and the achievable accuracy of the prediction. An attempt was made to link these two properties to fundamental characteristics of the wave energy conversion system, on the basis of the preliminary work presented in Fusco et al. (2010). From time-domain simulations of a number of floating cylinders over a variety of sea states, one parameter in particular was found to have the main influence (nearly proportional) on the required forecasting horizon for the achievement of a certain performance. Such a parameter is the main time constant of the non-causal transfer function for the calculation of the reference velocity, which depends only on the shape of the system's radiation resistance and is not shape-specific. Regrading the actual ability to predict, the system parameter highlighted was, as expected, the bandwidth of the excitation transfer function, which is, in general, a lowpass filter. Systems with smaller bandwidths allow for better predictions longer into the future when purely stochastic and regressive models are utilised. In this case, however, environmental parameters, such as bandwidth and resonance frequency of the wave system, also have an influence.

By comparing the required prediction horizons, between 10 and more than 60 seconds, and the actual predictability, between 0.5 and 1 wave period with more than $60 \%$ of accuracy (6 to 13 seconds for most sea states), one cannot fail to notice that in some cases the realisation of reactive control is impractical, unless a significantly better solution for the wave prediction problem is found. This is true, in theory, but there actually exists the possibility to properly shape the reference generation function, $H_{o p t}(\omega)$, by also considering at what frequencies the waves appear, such that the correspondent impulse response has a less noncausal behavior. An extreme example of such manipulation, resulting in a causal solution, is proposed in Fusco and Ringwood (2011).

\section{ACKNOWLEDGEMENTS}

The authors are grateful to the Irish Marine Institute for providing real sea observations.

\section{REFERENCES}

Babarit, A. and Clement, A. (2006). Optimal latching control of a wave energy device in regular and irregular waves. Applied Ocean Research, 28, 77-91.

Bacelli, G., Gilloteaux, J.C., and Ringwood, J. (2009). A predictive controller for a heaving buoy producing potable water. Proc. of the European Control Conference (ECC) 2009, Budapest, Hungary, 3755-3760.

Belmont, M., Horwood, J., Thurley, R., and Baker, J. (2006). Filters for linear sea-wave prediction. Ocean Engineering, 33, 2332-2351.

Cretel, J., Lewis, A.W., Lightbody, G., and Thomas, G.P. (2010). An application of model predictive control to a wave energy point absorber. Proc. Of the IFAC Conference on Control Methodologies and Technology for Energy Efficiency (CMTEE) 2010, Portugal.

Falnes, J. (2002). Ocean Waves and Oscillating Systems. Cambridge University Press.

Falnes, J. (2007). A review of wave-energy extraction. Marine Structures, 20, 285-201.

Fusco, F. and Ringwood, J. (2011). Suboptimal causal reactive control of wave energy converters using a second order system model. Proc. of the Intern. Society of Offshore and Polar Engineers (ISOPE), Maui, USA.

Fusco, F., Gilloteaux, J.C., and Ringwood, J. (2010). A Study on Prediction Requirements in time-domain Control of Wave Energy Converters. Proc. of Control Applications in Marine Systems (CAMS), Germany.

Fusco, F. and Ringwood, J. (2010). Short-Term Wave Forecasting for Real-Time Control of Wave Energy Converters. IEEE Trans. on Sustainable Energy, 1, no. 2, 99-106.

Hals, J., Falnes, J., and Moan, T. (2011). Constrained optimal control of a heaving buoy wave-energy converter. J. of Offshore Mechanics and Arctic Engineering, 133.

Korde, U.A. (2000). Control system applications in wave energy conversion. Proceedings of the OCEANS 2000 MTS/IEEE Conference and Exhibition, 3, 1817-24.

MacMartin, D. and Hall, S. (1991). Control of uncertain structures using an $H_{\infty}$ power flow approach. Journal of Guidance, 14, no. 3, 521-530.

Tedd, J. and Frigaard, P. (2007). Short term wave forecasting, using digital filters, for improved control of wave energy converters. Proc. of Int. Offshore and Polar Eng. (ISOPE) Conf., 388, 394.

Van Den Boom, H. (2009). Owme project makes technological breakthrough. Marin Report.

WAMIT Inc. (2008). Wamit. MA, USA, version 6.4. 